\title{
Future Home- A Review of Future House or Home with Security and Voice Controller
}

\author{
Satyendra Singh, Reetek Verma, Prashant Verma
}

\begin{abstract}
Home Automation using internet of things is an emerging technology and todays need. This technology offers new and exciting opportunities to increase the connectivity of appliances within the home for automation. Generally, Wi-Fi, Bluetooth, GSM modules or an Android app are used by the existing methods for controlling various home appliances. But the expansion of internet in the last decade, gives one the potential for controlling and automating various appliances through it in a comfortable and secure way. On the other hand, Artificial Intelligence is evolving as a technology for developing automatic systems that can perceive the environment, learn from environment, and can make decision using case based reasoning. The framework for taking real-time decision and automation for IoT is provided by artificial intelligence. AI has vision ability, knowledgebase, learning and decision-making ability, which makes it a better solution for automatic systems. This paper mainly aims to provide the user the facility to control their home appliances using internet and artificial intelligence, which will help them saving the electric power and human energy. The various appliances are connected to the sensor which is connected using Wireless Networks.
\end{abstract}

Index Terms - Home Automation, Internet of Things, Artificial Intelligence.

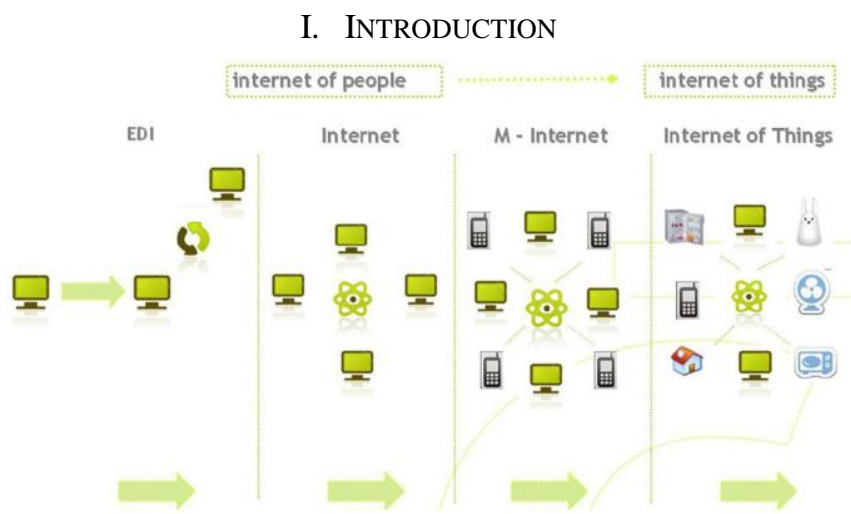

Fig. 1 Example of Internet Of Things (IOT)

An automated and networked home is "An automated and networked home is one in which every appliance can be remotely manage from any on the Internet with a simple Web browser". The general goal of the automatic-home[1] movement is to use networking technology to integrate the devices, appliances and services

Satyendra Singh, Department of Computer Science And Engineering, PSIT College Of Engineering, Kanpur City, India

Reetek Verma, Department of Computer Science And Engineering, PSIT College Of Engineering, Kanpur City, India.

Prashant Verma, Department of Computer Science And Engineering, PSIT College Of Engineering, Kanpur City, India. found in homes so that the entire domestic living space can be controlled centrally or remotely. Automation is a technique, method, or system operating or controlling a process by electronic devices with reducing human involvement to a minimum. The fundamental of building an automation system for an office or home is increasing day-by-day with numerous benefits. Industrialist and researchers are working to build efficient and Affordability automatic systems to monitor and control different machines like lights, fans, AC based on the Requirement Automation makes not only an efficient but also an economical use of the electricity and water and reduces much of the wastage. It is impossible for someone to predict that IoT would come into existence in a very short period. IoT is one of the promising technologies used to control objects connected to internet through IP address. In Real Time Application[2] such as smart home, smart cities, smart grids and intelligent transportation, it is widely used. It has also expanded its features into medical fields such as instruments and many more. Smart home is one in which various electronic and electric appliances are wired up to a central computer control system so that they can either be switched on and off at certain times. Cloud computing is a rapidly growing technology where're sources such as storage devices, platform and applications are shared over the internet and is widely

used by multiple users in small and medium business. Cloud services can be provided and delivered remotely by vendors such as Amazon or Microsoft as "public clouds" [4], or the resources are designed, installed, monitored and controlled internally as "private clouds"[7]. Cloud data retrieval is an important service to be considered as certain specific data files the users are interested during a given session must be retrieved in an efficient way and quickly. In this project we are connecting the various sensors and devices to Raspberry $\mathrm{Pi}$ which is connected with IFTTT [3] and the devices are operated as per the voice commands given through Google Assistant.

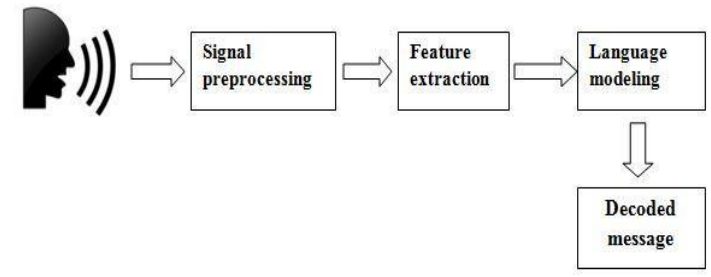

Fig. 2 Example of Speech to signal processing 


\section{MOTIVATION}

During the past few years, internet was known as a big mass that we can acquire data from. Embedding mobile transceivers to everyday items and gadgets enabled new forms of bi-directional communication between people with other people, and people with things. That paradigm, known as Internet of Things, that was first introduced in 1998 by Kevin Ashton, has received recently more attention in the academia and industry, and this would add a new dimension to the world of Information and communication technology. While that paradigm is growing and have high positive impact on many aspects of our lives, challenging issues arise, that should be considered and addressed. The central issues are guaranteeing security and privacy of users and their data. Another issue is fully achieving smartness of interconnected devices by enabling their interaction. Exchanging data and autonomous behavior is the key to achieving the latter .

IoT has different definitions from different perspectives, however, they all revolve around "things" generally, collecting, exchanging and communicating data with each other's and with people through the "internet". IoT helps in decision making and au- to mating almost everything around us. The smarter life IoT vision promises in the near future through various applications, made smart Home Automation actually possible, starting from basically monitoring different parts of home, to actually controlling them. Integration of IoT and Home Automation, made it possible to monitor and control homes from different parts of the world. Some examples of applications to this are: controlling and setting the desired temperature of the house before arriving home, turning on/off the lights of a room and setting its intensity, running washing machine while the person is at work, leakage or smoke detection and notification, monitoring home through surveillance camera or car inside the house while the person is away, or remote central locking, and many other applications.

As the field of Home Automation through IoT is a wide application in a very wide and challenging field due to the reasons mentioned in the previous paragraphs, I chose to work on that field as part of this thesis, specifically in maintaining and ensuring security and safety inside home.

Today wherever we go, we hear a term called "IOT" which is creating more buzz in the list of emerging technologies in embraced by many big brands consumers, and startups paying a lots of innovation in technology.

Nowadays, whether a person is at home or in office or in business, he/she needs to reduce their time in doing routine within the time frame available: managing office activities according to the schedules. The solution for the above comfort is automation and networking of people and devices.

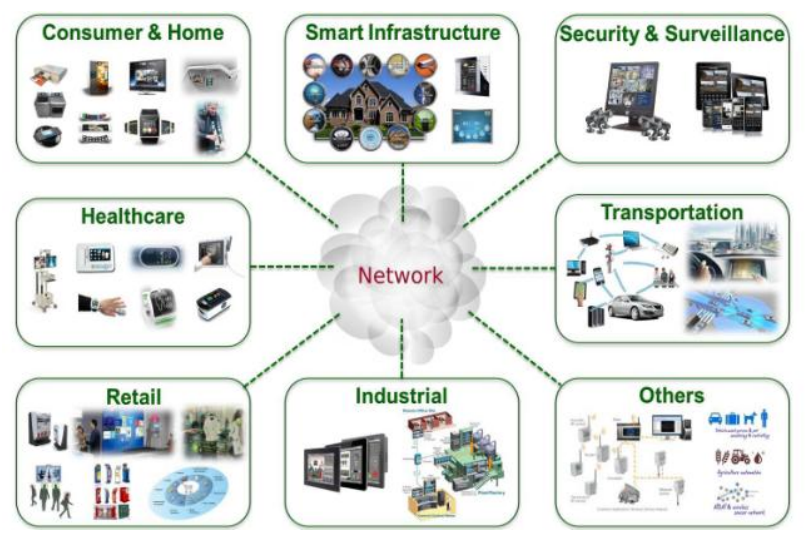

Fig. 3 Areas Of Internet Of Things

\section{AIM AND OBJECTIVE}

This paper addresses an IoT software-based approach on the field of Home Automation. Common use-cases include measuring home conditions, controlling home appliances and controlling home access through RFID cards as an example and windows through servo locks. However, the main focus of this paper is to maximize the security of homes through IoT. More specifically, monitoring and controlling servo door locks, door sensors, surveillance cameras, surveillance car and smoke detectors, which helps ensuring and maximizing safety and security of homes.

A user has the following features of this Future home in which a person can:-

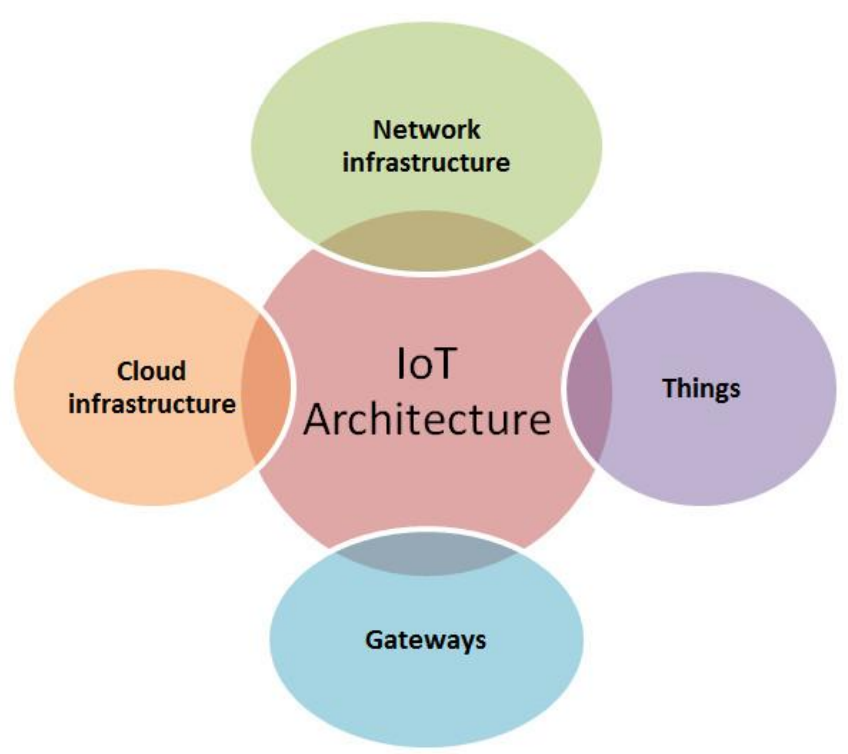

Fig. 4 IOT Structure

1. Can turn on or off the lights using your voice

2. Can lock and unlock the gate of your doors using RFID

3. This Gate is controlled with the help of Servo Motor.

4. Can monitor the main gate with the help of camera.

5. Can park your car without exiting from it.

6. Advanced Security feature with laser security in home.

7. Solar tracker device which follows the sun.

8. Can Ring the doorbell without touching it.

9. Can On/off the garden light with the increasing and decreasing the intensity of light. 
10. Can open the main gate when the authorized person is in front of it.

\section{A. Voice control using IFTTT}

The full form of IFTTT[3] is If this then that IFTTT is both a website and a mobile app. The free service launched in 2010 with the following slogan put the internet to work for you. IFTTT basically works as interface between the nodeMCU and the appliances that are attached to nodeMCU We used this technology in order to connect all our appliances together so that we can control all with the help of our smart phone .we came to this solution because of the people who are old age and who are unable to unable to move properly and also for blind person ,they just simply have to say some simple words through the google assistance to function all their home appliances we have following steps to connect the appliances to work for you.

Steps: Install and Configure Blynk App and then we login into it and then we give the project name and select hardware as nodeMCU and connection type Wi-Fi then a AUTH code will be sent to our Gmail id ,we copy that AUTH code to log in to the website after that we come to the applet making process in this we click on the this search for google assistance and then click to connect then we are given a trigger which simply mean when we say some phrase it will perform some work now to do some work we connect through the web hook which is a tool that will send the web request to the Blynk app which will send the command to nodeMCU and node MCU controls the appliances .Now to check this open our google assistance and say that phrase which we made at the time of applet making process and it works as controlling the appliances .we also have to also upload the code for the node MCU in Arduino IDE.

B. Solar Tracker [5] which tracks the sun

A solar panel is a made up of Photovoltaic cells which absorbs the sun light and convert it into electricity. If we fix a solar panel to rooftops and open area than the quantity of electricity which is generated is less as researched.

In the model view we use two servo motors by which our model is a dual axis rotator and we put LDR(light dependent resistors) for the rotation and calculation of the light intensity.

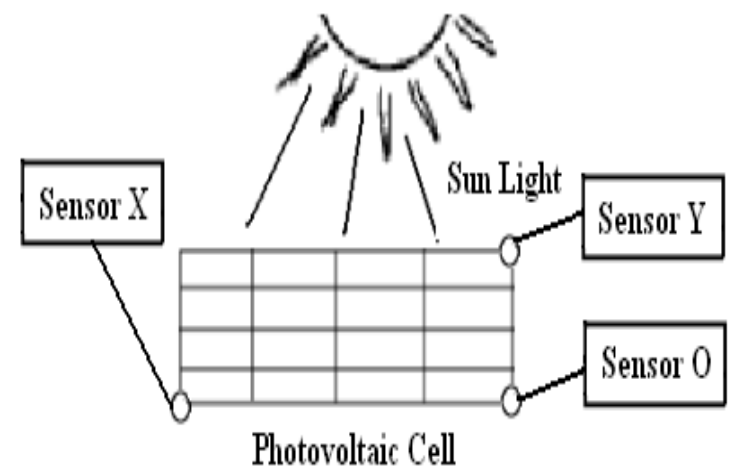

Fig. 5 Solar Tracker
TABLE 1

\begin{tabular}{|c|c|c|}
\hline TIME & $\begin{array}{l}\text { CURRENT USING } \\
\text { FIXED } \\
\text { PHOTOVOLTAIC } \\
\text { CELL } \\
\text { (AMP) }\end{array}$ & $\begin{array}{l}\text { CURRENT USING } \\
\text { MOVABLE SOLAR } \\
\text { PHOTOVOLTAIC } \\
\text { CELL } \\
\text { (AMP) }\end{array}$ \\
\hline 8:00 AM & 0.42 & 0.85 \\
\hline 10:00 AM & 1.3 & 1.82 \\
\hline 12:00 AM & 1.73 & 1.93 \\
\hline 2:00 PM & 1.83 & 1.97 \\
\hline 6:00 PM & 1.18 & 1.93 \\
\hline 4:00 PM & 0.09 & 1.36 \\
\hline & $\mathbf{6 . 9 3}$ & \\
\hline
\end{tabular}

The efficiency of the proposed system can be calculated using the equation (1):

Efficiency $=(11.36-6.93) * 100=(1)$

It seems that the efficiency of the proposed system can be increased around $64 \%$ on a summer sunny day. In addition, the proposed system consumes little power to turn the PV panel using a small stepper motor instead of using large panelwhich consumes larger amount of power

We have seen the voltage generated by fixed panel and rotation panel and we have seen the high generation of voltage in rotating panel.

That's why we use this model for the main backup in the home. 


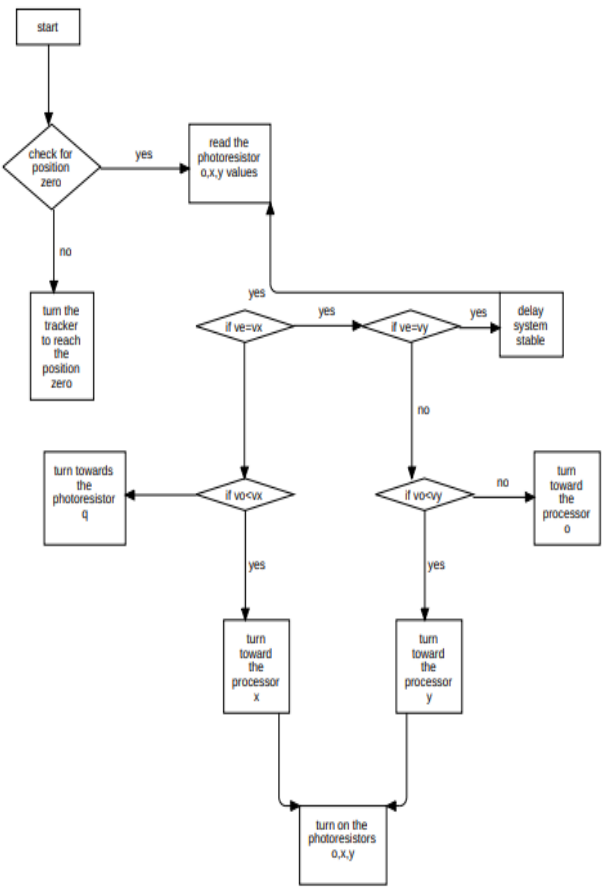

Fig. 6 Data Flow Diagram

\section{RFID Gate Lock}

RFID[8] is used for Radio-frequency identification and this used the electromagnetic fields which automatically identify and track the device with the help of RFID cards or tags. This tag or cards contains electronically-stored information which and this information can be useful or can be fetched when this cards is bring near to the RFID receiver. In this we install a RFID reader for unlocking the gates of the home. For Opening and closing the gate install a Servo Motor and the properties of Servo Motor is it always moves the 180 degree or we can say that it is a rotary actuator. In this we uses two servo motors and both the servos are identified with the help of RFID cards.

We have two cards the slave and the master card. The Master card is for the study room and the slave card is for the bedroom. We register the Hexadecimal value in the ATMEGA 328P microcontroller with the two servos If we bring the card in front of RFID reader then the gate will Open and this Gate will automatically close after the 10 seconds.

\section{D.Can on/off the garden light with the increasing and decreasing the intensity of light.}

In this feature turning on/off the garden light with the help of intensity of light. The LED light is made up off light dependency register (LDR) [9].when the intensity of the light is low the garden light will automatically turn on. If the light intensity is good the light will automatically turn off. We made this for the energy saving as well as low cost provide us.

LDR-> When the light level decreases, the resistance of the LDR increases. As this resistance increases in relation to the other Resistor, which has a fixed resistance, it causes the voltage dropped across the LDR to also increase.

When this voltage is large enough $(0.7 \mathrm{~V}$ for a typical NPN Transistor), it will cause the Transistor to turn on. We can turn on this light by using $5 \mathrm{v}$ to $12 \mathrm{v}$ electrical appliances.

The value of the fixed resistor will depend on the LDR used, the transistor used and the supply voltage.

\section{E. can open the main gate when authorize person is in front it.}

In this feature we will provide the entry to only authorized person. When the person is in front of main gate the person will get identified through the camera which is placed on the house wall. If the person is known we will provide the entry into the house. For making the automatic gate we uses two servo motor, laser light And IR sensors[10].

In this we will use Digital image processing[6] (DIP) for detecting the person. Procedure when the person stands in front of gate the person picture is send to the administrator of the house and the authorization is done. The gate will open automatically. It will provide us security to our house member.

Servo motor->A servomotor is a rotary actuator or linear actuator that allows for precise control of angular or linear position, velocity and acceleration. It consists of a suitable motor coupled to a sensor for position feedback.

\section{REFERENCES}

[1] MUHAMMAD ASADULLAH, KHALIL ULLAH, "SMART HOME AUTOMATION SYSTEM USING BLUETOOTH TECHNOLOGY", INNOVATIONS IN ELECTRICAL ENGINEERING AND COMPUTATIONAL TECHNOLOGIES (ICIEECT) 2017 INTERNATIONAL CONFERENCE ON, PP. 1-6, 2017.

[2] J. Erdelyi, P. Čičák, "Survey on Communication in Internet of Things Environment", Emerging eLearning Technologies and Applications (ICETA) 2018 16th International Conference on, pp. 149-156, 2018.

[3] W. Wayt Gibb IEEE Spectrum Volume: 53 , Issue: 1 , January 2016

[4] Soumen Moulik, Sudip Misra, Mohammad S. Obaidat, "Smart-Evac: Big Data-Based Decision Making for Emergency Evacuation", Cloud Computing IEEE, vol. 2, no. 3, pp. 58-65, 2015.

[5] Debabrata Mazumdar, Dola Sinha, Surojit Panja, Dipak Kumar Dhak, "Design of LQR controller for solar tracking system", Electrical Computer and Communication Technologies (ICECCT) 2015 IEEE International Conference on, pp. 1-5, 2015.

[6] Peter Seitz, Peter Ruegsegger, "Fast Contour Detection Algorithm for High Precision Quantitative CT", Medical Imaging IEEE Transactions on, vol. 2, no. 3, pp. 136-141, 1983.

[7] Hongbin Lu, Mark Shtern, Bradley Simmons, Michael Smit, Marin Litoiu, "Pattern-Based Deployment Service.for Next Generation Clouds", Services (SERVICES) 2013 IEEE Ninth World Congress on, pp. 464-471, 2013

[8] M.A. Ziai, John C. Batchelor, "Smart radio-frequency identification tag for diaper moisture detection", , vol. 2, no. 1, pp. 18-21, 2015.

[9] D. F. Silva, D. A. Avalos, "Light Dependent Resistance as a Sensor in Spectroscopy Setups Using Pulsed Light and Compared with Electret Microphones", MDPI 2006, ISSN 1424-8220.

[10] B.R. HUNT DIGITAL IMAGE PROCESSING Volume: 63, Issue: 4, April 1975 